\title{
Negative Pressure Pulmonary Edema as a Cause of Diffuse Alveolar Hemorrhage in the Newborn
}

\author{
Alejandro Donoso ${ }^{1}$ Gianfranco Tomarelli ${ }^{1}$ Daniela Arriagada ${ }^{1}$ \\ 1 Pediatric Intensive Care Unit, Hospital Clínico Metropolitano La \\ Florida, Santiago, Chile

\begin{abstract}
Address for correspondence Alejandro Donoso Fuentes, Pediatric Intensive Care Unit, Hospital Clínico Metropolitano La Florida, Froilán Roa 6542, Santiago, Chile (e-mail: adonosofuentes@gmail.com).
\end{abstract}

J Child Sci 2020;10:e212-e214.

Abstract
Keywords
- pulmonary edema
- diffuse alveolar
hemorrhage
- newborn

Negative pressure pulmonary edema (NPPE) is a rare entity that can become life threatening. Its development in neonates is very rare, and its presentation as alveolar hemorrhage is uncommon. We report a case of a newborn 23 days old, previously healthy, who presented an episode of choking during breastfeeding. This progressed to acute respiratory failure due to diffuse alveolar hemorrhage. A few hours after admission, the newborn developed refractory hypoxemia, requiring high-frequency oscillatory ventilation and nitric oxide therapy for 24 hours. NPPE was postulated as a diagnosis of exclusion. The newborn recovered completely. NPPE should always be considered in a case with recent obstruction of the upper airway, even in unusual age groups. Sometimes it can manifest as alveolar hemorrhage.

\section{Introduction}

Negative pressure pulmonary edema (NPPE) is a rare and probably underdiagnosed complication. ${ }^{1}$ It is an infrequent cause of diffuse alveolar hemorrhage in children, but its development can be life threatening. Upper airway obstruction produces a marked inspiratory effort, which generates an excessive intrathoracic negative pressure, causing pulmonary edema and, exceptionally, hemoptysis. ${ }^{2}$

Its development in newborns is infrequent; anatomical and physiological characteristics, such as greater distensibility and lower development of thoracic musculature, make it difficult to generate extreme intra-alveolar negative pressures.

We report a 23-day-old newborn, previously healthy, who presented with an episode of choking during breastfeeding, causing diffuse alveolar hemorrhage secondary to NPPE. The newborn made a full recovery.

\section{Case Report}

We present a 23-day-old male patient, term newborn, previously healthy, who is first birth of an 18-year-old mother with a late obstetric presentation. While breastfeeding, he suddenly presented respiratory distress, oral cyanosis, and cough, requiring immediate referral to emergency services. Upon admission, 15 minutes after the event, he presented a temperature of $36.6^{\circ} \mathrm{C}$, oxygen saturation $86 \%$, and capillary glycemia $154 \mathrm{mg} / \mathrm{dL}$. Physical examination showed general paleness, poor perfusion, and polypnea with significant use of intercostal muscles. No external lesions were evidenced in the oral cavity or in the thorax. The chest radiograph ( - Fig. 1) showed radiopacity of both lung fields.

Laboratory tests highlighted leukocytes $9,900 / \mathrm{mm}^{3}$, platelets $331,000 / \mathrm{mm}^{3}$, hemoglobin at $13.8 \mathrm{~g} / \mathrm{dL}$, C-reactive protein $0.02 \mathrm{mg} / \mathrm{dL}$ (normal value $(\mathrm{NV})<1 \mathrm{mg} / \mathrm{dL}$ ), prothrombin time (PT) 81\%, activated partial thromboplastin time (aPTT) 38.6 ("NV 26-40"), venous gases: $\mathrm{pH} 7.16$, partial pressure of carbon dioxide $69 \mathrm{~mm} \mathrm{Hg}$, base excess (BE) -6. He was hospitalized in our intensive care unit. During endotracheal intubation, we observed bleeding from the infraglottic region.

The echocardiogram showed no anatomic abnormalities, with indirect signs of moderate pulmonary hypertension. Electrocardiogram and troponins were normal.

Initial management included mechanical ventilation, vasoactive support, antibiotic therapy (ampicillin and cefotaxime), sedation (midazolam and fentanyl), and neuromuscular block received

August 3, 2020

accepted after revision

October 14, 2020
DOI https://doi.org/

10.1055/s-0040-1721142. ISSN 2474-5871.
Copyright @ 2020 Georg Thieme Verlag License terms KG Rüdigerstraße 14, 70469 Stuttgart, Germany

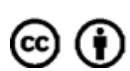




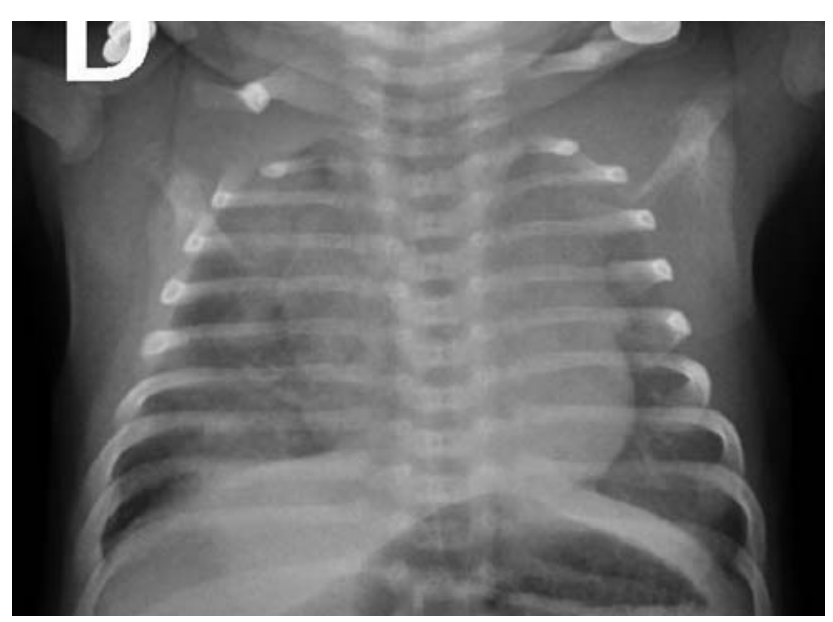

Fig. 1 Chest radiograph showing the radiopacity of both lung fields.

(vecuronium) as a continuous infusion. He also required a transfusion of red blood cells and fresh frozen plasma.

Computed tomography (CT) of chest, at 6 hours of admission, showed confluent bilateral alveolar infiltrate, compatible with diffuse alveolar hemorrhage (-Fig. 2). No anatomical malformations were observed.

After 6 hours, he was connected to high-frequency oscillatory ventilation (PMVA $25 \mathrm{~cm} \mathrm{H}_{2} \mathrm{O}$ ) and inhaled nitric oxide $(20 \mathrm{ppm})$ due to refractory hypoxemia (partial pressure of oxygen/fraction of inspired oxygen: $80 \mathrm{~mm} \mathrm{Hg}$ ), with clinical and laboratory improvement in the next 24 hours, continuing with conventional mechanical ventilation for 36 hours. Supplemental oxygen was ceased on the fifth day of hospitalization.

The following etiological studies were performed: blood cultures, tracheal aspirate culture, cerebrospinal fluid culture, test for respiratory viruses, polymerase chain reaction for Pneumocystis jiroveci, serology for cytomegalovirus, enzyme-linked immunosorbent assay test for human immunodeficiency virus, immunological tests, and study for inborn

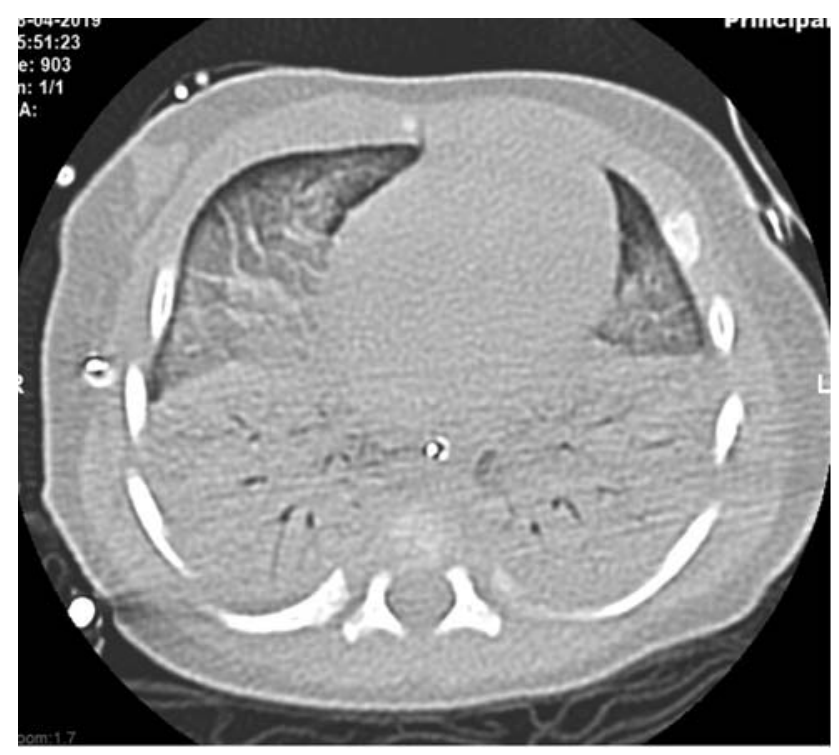

Fig. 2 Computed tomography of chest with signs of diffuse alveolar hemorrhage. errors of metabolism. All of them were negative. In the context of refractory hypoxemia, brain CT and electroencephalogram were performed, resulting both normal.

He received antibiotic therapy for 10 days. Nocturnal oxygen saturation monitoring and suction study were performed prior to discharge and both were normal. Social service shows no evidence of problems. We concluded that this case was an episode of alveolar hemorrhage due to NPPE, caused by an accidental event of choking while the child was breastfeeding. Patient follow-up at 12 months showed a normal chest $\mathrm{X}$-ray and good weight increase, without new bleeding episodes.

\section{Discussion}

Choking is a well-described entity in neonatal patients during their hospital stay in the immediate postpartum period $^{3}$ as well as in their homes. ${ }^{4}$ This may present with hypoxic-ischemic symptoms after a respiratory arrest.

NPPE usually presents in adult patients who develop postextubation laryngospasm as a complication after receiving anesthesia. In pediatric patients, the development of NPPE is unusual, being the first case described by Travis et al. ${ }^{5}$

Currently, there are several articles that indicate a wide spectrum of pathologies associated with upper airway obstruction, but most of them in older patients. ${ }^{6,7}$

NPPE-associated pulmonary hemorrhage was reported by Schwartz et al. $^{8}$ It is an entity, often underdiagnosed and potentially lethal.

The triggering mechanism of NPPE is an excessive intrathoracic negative pressure caused by forced inspiration against total or partial airway obstruction. This causes a decrease in right atrial pressure and, secondarily, an increase in venous return to the right heart, causing an increase in hydrostatic pressure in the pulmonary vasculature, which promotes the development of alveolar edema. Also, the excessive intrapleural negative pressure is transmitted to the pulmonary interstitium, thus contributing to the development of interstitial and alveolar edema. Simultaneously, there is an increase in the left ventricular afterload (greater transmural pressure), as well as a decrease in the distensibility of the left ventricle due to the displacement of the interventricular septum, secondary to a greater venous return, which causes elevation of the left ventricular diastolic pressure and a higher microvascular hydrostatic pressure. Thus, the elevation of the pulmonary capillary transmural pressure favors fluid transudation into the interstitial and alveolar space causing pulmonary edema. ${ }^{2}$ NPPE should be considered as a clinical spectrum, where the greater transmural capillary pressure causes circumferential stress on the wall, resulting in stress failure of the epithelial and endothelial surface. Due to a slight increase in transmural pressure, exudative edema occurs and, in some cases, microhemorrhages. The existence of extremely high capillary transmural pressures causes rupture of the membrane with the consequent alveolar hemorrhage (seal of capillary stress failure). 
The negative pressure threshold that causes severe disruption of the pulmonary microvasculature has not been established for humans, but is probably high. ${ }^{6}$

The maximum inspiratory pressures are very high in neonates, compared with adults, because the radius of the curvature of the rib cage, diaphragm, and abdomen is small (Laplace's law). Esophageal pressures of up to $-50 \mathrm{~cm} \mathrm{H}_{2} \mathrm{O}$ (range: -28 to $-105 \mathrm{~cm} \mathrm{H}_{2} \mathrm{O}$ ) have been recorded at birth. ${ }^{9}$

In summary, NPPE is the result of three pathophysiological processes: excessive intrathoracic negative pressure, increased pulmonary capillary hydrostatic pressure and mechanical stress on the alveolar-capillary membrane, which causes its rupture.

In addition, the existence of hypoxia, hypercapnia, and secondary hyperadrenergic status can increase blood flow and pulmonary vascular resistance as well as cause myocardial dysfunction. ${ }^{2}$

NPPE diagnosis is clinical and is based primarily on the history of a recent obstructive event of the upper airway. In this way, the precipitating incident will be the key of the diagnosis.

In the physical examination, the presence of tachycardia, tachypnea, crackles, and oxygen desaturation stands out. Symptoms usually develop immediately. Considering the above, it is important to maintain adequate monitoring of patients with risk factors.

The radiological findings show images compatible with alveolar edema and normal cardiac size. Occasionally, cases of diffuse alveolar hemorrhage secondary to NPPE have been described. ${ }^{10}$

NPPE with hemorrhage is a diagnosis of exclusion. The existence of cardiogenic edema, hemorrhagic pneumonia, septic shock with coagulopathy, convulsive status, fatty acid oxidation defects, blood aspiration from the airway, and chest contusion should always be ruled out, as was the case here reported.

Its treatment is supported by oxygen therapy. Sometimes the use of noninvasive mechanical ventilation is beneficial. Fluid restriction such as steroid and diuretic use is controversial. ${ }^{2}$ If the NPPE is treated early, it is usually a self-limited and reversible condition, with a favorable prognosis.

This case stands out for the age of presentation and for the development of alveolar hemorrhage. We think that NPPE was caused by an accidental episode of mechanical suffocation that was not opportunely perceived while the child was breastfeeding. In this way, it seems to us that, although the duration of the obstructive episode or the inspiratory force made by the patient can never be specified, the sudden onset of symptoms, the absence of other causes ruled out by relevant examinations, the exclusive existence of hypoxemia, the radiological findings, and the satisfactory therapeutic response as well as its favorable long-term evolution strongly support the diagnosis of this entity. A limitation of our patient's etiological study was the nonperformance of bronchoscopy with bronchoalveolar lavage considering his clinical condition, which would have allowed us to rule out the existence of a tracheobronchial lesion, and the absence of hemosiderophages would have confirmed the acute nature of the hemorrhage.

Considering the number of victims of suffocation in the lactation period and the scarcity of similar reports, we believe that the rush of the consultation and an optimal resuscitation allowed the manifestation of this pathophysiological phenomenon in our patient.

Conflict of Interest

None declared.

\section{References}

1 McConkey P. Airway bleeding in negative-pressure pulmonary edema. Anesthesiology 2001;95(01):272

2 Lemyze M, Mallat J. Understanding negative pressure pulmonary edema. Intensive Care Med 2014;40(08):1140-1143

3 Thach BT. Deaths and near deaths of healthy newborn infants while bed sharing on maternity wards. J Perinatol 2014;34(04): 275-279

4 Hayman RM, McDonald G, Baker NJ, Mitchell EA, Dalziel SR. Infant suffocation in place of sleep: New Zealand national data 20022009. Arch Dis Child 2015;100(07):610-614

5 Travis KW, Todres ID, Shannon DC. Pulmonary edema associated with croup and epiglottitis. Pediatrics 1977;59(05):695-698

6 Contou D, Voiriot G, Djibré M, Labbé V, Fartoukh M, Parrot A. Clinical features of patients with diffuse alveolar hemorrhage due to negative-pressure pulmonary edema. Lung 2017;195(04): 477-487

7 Chen J, Wang S, Ma H, et al. [Clinical analysis of 4 children with negative pressure pulmonary edema]. Zhonghua Er Ke Za Zhi 2014;52(02):122-127

8 Schwartz DR, Maroo A, Malhotra A, Kesselman H. Negative pressure pulmonary hemorrhage. Chest 1999;115(04): 1194-1197

9 Vyas H, Field D, Milner AD, Hopkin IE. Determinants of the first inspiratory volume and functional residual capacity at birth. Pediatr Pulmonol 1986;2(04):189-193

10 Dolinski SY, MacGregor DA, Scuderi PE. Pulmonary hemorrhage associated with negative-pressure pulmonary edema. Anesthesiology 2000;93(03):888-890 